\title{
Probabilistic Social Sequential Model for Tour Recommendation
}

\author{
Vineeth Rakesh \\ Wayne State University \\ Detroit, MI - 48202 \\ vineethrakesh@wayne.edu \\ Alexander Kotov \\ Wayne State University \\ Detroit, MI - 48202 \\ kotov@wayne.edu
}

\author{
Niranjan Jadhav \\ Wayne State University \\ Detroit, MI - 48202 \\ niranjan.jadhav@wayne.edu \\ Chandan K. Reddy \\ Virginia Tech \\ Arlington, VA - 22203 \\ reddy@cs.vt.edu
}

\begin{abstract}
The pervasive growth of location-based services such as Foursquare and Yelp has enabled researchers to incorporate better personalization into recommendation models by leveraging the geo-temporal breadcrumbs left by a plethora of travelers. In this paper, we explore Travel path recommendation, which is one of the applications of intelligent urban navigation that aims in recommending sequence of point of interest (POIs) to tourists. Currently, travelers rely on a tedious and time-consuming process of searching the web, browsing through websites such as Trip Advisor, and reading travel blogs to compile an itinerary. On the other hand, people who do not plan ahead of their trip find it extremely difficult to do this in real-time since there are no automated systems that can provide personalized itinerary for travelers. To tackle this problem, we propose a tour recommendation model that uses a probabilistic generative framework to incorporate user's categorical preference, influence from their social circle, the dynamic travel transitions (or patterns) and the popularity of venues to recommend sequence of POIs for tourists. Through comprehensive experiments over a rich dataset of travel patterns from Foursquare, we show that our model is capable of outperforming the state-of-the-art probabilistic tour recommendation model by providing contextual and meaningful recommendation for travelers.
\end{abstract}

\section{Categories and Subject Descriptors}

H.2.8 [Database Management]: Database applicationsData Mining; I.2.6 [Artificial Intelligence]: Learning; H.3.3 [Information Search and Retrieval]: Information filtering

\section{Keywords}

Recommender Systems; Probabilistic Generative Models; Topic Models; Geo-location; Foursquare; Social Media

Permission to make digital or hard copies of all or part of this work for personal or classroom use is granted without fee provided that copies are not made or distributed for profit or commercial advantage and that copies bear this notice and the full citation on the first page. Copyrights for components of this work owned by others than ACM must be honored. Abstracting with credit is permitted. To copy otherwise, or republish, to post on servers or to redistribute to lists, requires prior specific permission and/or a fee. Request permissions from permissions@ acm.org.

WSDM 2017, February 06-10, 2017, Cambridge, United Kingdom

(c) 2017 ACM. ISBN 978-1-4503-4675-7/17/02 . .\$15.00

DOI: http://dx.doi.org/10.1145/3018661.3018711

\section{INTRODUCTION}

Tour recommendation has become a new trend in the field of intelligent urban navigation. The dramatic increase in the amount of publicly available check-in data has generated substantial interest among different research communities to work on this problem. Different from conventional way of recommending independent venues, the objective of tour recommendation is to suggest a sequence of points of interest (POIs) that will serve as travel itineraries to users. Tour recommendation is more challenging than the conventional one due to two main reasons. First, since most users are not native to their tour destination (i.e. users are tourists), the check-in information of these users is extremely sparse. Therefore, using simple collaborative filtering based techniques will yield poor recommendation results. To overcome this problem, it is crucial to learn the topical preference of users from their historical check-ins and incorporate them as content-based features to create a hybrid recommendation model. Second, many researchers have shown that human mobility exhibits a strong temporal pattern $[31,5]$. Unlike conventional recommendation, where POI suggestions are made in a disjoint manner, these temporal features play a critical role in determining the next check-in spot when suggesting a sequence of POIs for travelers.

In addition to the above-mentioned traits of tour recommendation, LBSNs such as Foursquare and Yelp enable travelers to communicate with other fellow travelers, interact with the residents from their tour destination and add other users to their social network of friends to make an informed choice about the travel destination. Therefore, it is essential to utilize the social network of travelers as implicit meta-data information to create a robust recommendation framework. Finally, travelers have limited duration of stay and they tend to prefer venues that are popular and well rated. Consequently, it is important to factor-in the POI-specific characteristics such as geographical distance between venues and their popularity. We illustrate the abovementioned traits using a toy example in Figure 1 that depicts a tourist who begins his journey at New York's JFK airport. Let us assume that the tourist plans to stay for just 1 day and his topical interests are nature and history museums. Based on the time constraints and topical preference, one logical sequence would be to start with Prospect park, which is geo-graphically closer to his current location, and suggest venues such as the Old Stone house, Botanical gar- 
den and Brooklyn bridge. When compared to the POIs in Manhattan, although these locations are not extremely popular, they are well-rated and more importantly they match the topical interests of the user. Another option is to recommend the set of POIs in route 2. Contrary to the sequence in route 1 , the venues in route 2 are extremely popular, but they do not exactly match with the user's topical interest. This is just one scenario; as we can see, there are multiple travel routes. Nonetheless, the POIs suggested by a good recommendation system should be a blend of geographical distance, personal choice of the user, social preference of the user's community and popularity of the venues. To achieve this, in this paper, we propose a social sequential tour rec-

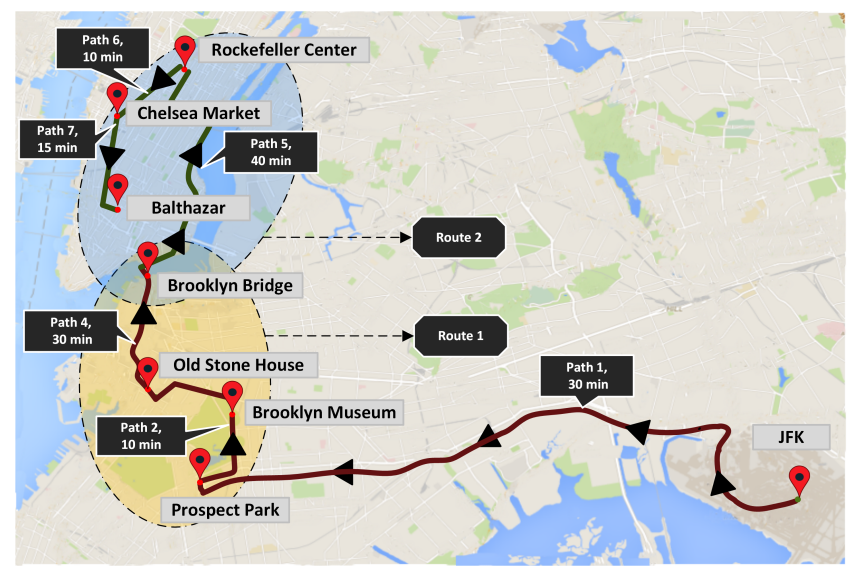

Figure 1: Travel pattern of a tourist who is interested in historical sites and nature.

ommendation model, abbreviated as SSTREC, that aims at providing personalized POI recommendations for travelers. Inspired by several state-of-the-art generative models [26, $24,23]$, we create a probabilistic framework that incorporates a multitude of features such as (a) the topical preference of users from their historical check-ins, (b) their friend's choice from the social network of travelers, (c) the sequential visiting patterns of travelers, and (d) the trending popularity of venues, into a unified supervised topic model to make effective suggestions of POI sequences.

The rest of this paper is structured as follows. We begin by providing some statistical insights about the behavior of travelers in Section 2. In Section 3, we introduce the proposed SSTREC model and the generative process. The description of the model is followed by the details of Gibbs sampling and the derivation of parameters in Section 4. We also explain the algorithm for creating POI sequences in this section. The data collection methodology and the results of our experiments are discussed in Section 5. Finally, we review the related works on location recommendation in Section 6 and conclude our paper in Section 7.

\section{LEARNING TRAVELER BEHAVIOR}

Before designing a recommendation model for travelers, it is important to understand their check-in behavior. In other words, we try to answer the question "What motivates travelers to visit a POI?" from four different perspectives namely: (a) sequential travel patterns, (b) topical interest of users, (c) impact of users' social circle and (d) popularity of POIs.
Impact of Travel Sequence: One of the main goals of this paper is to incorporate Markovian relationships between POIs into our recommendation model. Therefore, the first step is to understand the nature of decision making of travelers. In particular, we are interested in determining whether they follow sequential patterns when traveling or visit POIs randomly. To answer this question, we employ hypothesis testing and perform the following steps: (1) obtain the global travel pattern by calculating conditional probabilities of traveling from the source venue $X$ to target venue $Y$ for all POI combinations; (2) for each user, obtain the top 10 ranked global travel patterns that correspond to his POI visits. If the user has pursued at least $50 \%$ of the POI sequences from this global pattern, categorize him as followed, if not, categorize as not followed; (3) randomly sample 100 travelers for 1,000 iterations and count the number of users who followed and those who did not. The result of this experiment is shown in Figure 2(a), where we notice that the median number of users who adhere to a travel pattern are higher than those who do not. To test the significance of this result, we set our null hypothesis $\mathrm{H}_{0}$ as: "the average number of users who follow the sequence is same as those who don't". By applying a two-sample t-test, the null hypothesis was rejected with a significance value of $2.2 \times 10^{-16}$ thereby concluding that the majority of travelers visit POIs sequentially.
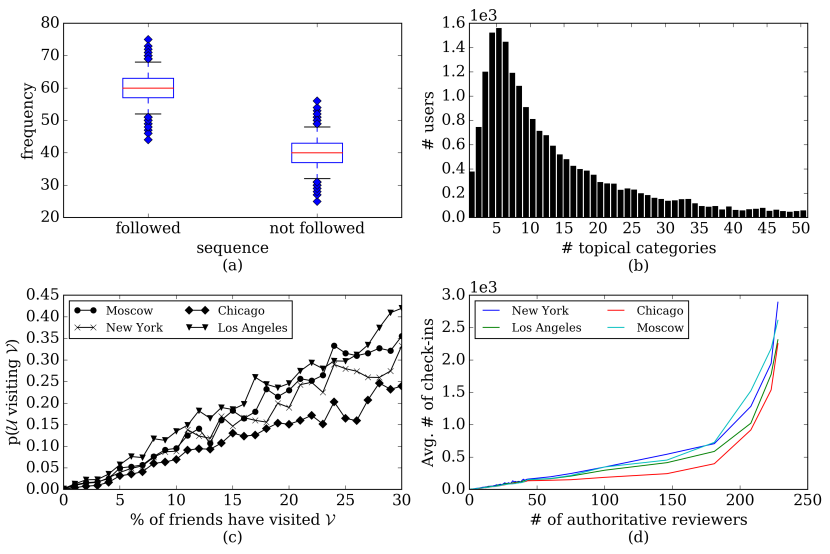

Figure 2: Behavior of travelers: (a) Variation in the distribution of follower versus non-followers; (b) Topical composition of tourists; (c) Impact of social circle on check-in behavior of tourists; (d) Influence of prominent reviewers over user check-ins.

Topical Composition: The decision to visit a landmark certainly depends on the topical interest of travelers. Nonetheless, to understand the topical variability of user interests, we extract the POI categories from their check-in history and plot the histogram of the topical compositions in Figure 2(b), where the $\mathrm{x}$-axis is the number of unique topical categories liked by the travelers. In general, we see that the travelers are interested in multiple topical categories of POIs. However, a majority of them are restricted to about 5-10 categories and people who are interested in over 30 topical categories are extremely few in number.

Impact from the Social Circle: For every traveler $u \in U$ in our dataset, we obtain their list of friends $F_{u}$ using 
the Foursquare $\mathrm{API}^{1}$. We then calculate the percentage of friends who have visited a location $v \in V$, and the probability of this user $u$ to visit $v$. The outcome of this analysis is depicted in Figure 2(c), which shows that the probability of a user visiting a POI increases as the cardinality of set $F_{u}$ get larger. In other words, the social circle of a user plays an important role in influencing the check-in habits of travelers.

Presence of Prominent Reviewers: Reviewers play a critical role in attracting tourists; therefore, we also investigate whether check-ins are influenced by POI reviews by authoritative reviewers. Consequently, for every POI, we calculate the number of authoritative users based on the upvotes of their tips (or reviews) and plot it against the number of check-ins in Figure 2(d). This plot indicates that POIs having large number of such authoritative users have the potential to attract many tourists.

\section{PROPOSED SSTREC MODEL}

In this section, we introduce SSTREC, a probabilistic generative model for recommending POIs for travelers. Our model is designed to capture the following behavioral traits of tourists: (1) traveling habits of users exhibit a strong sequential pattern, where the selection of a POI is dependent on the previously visited POI. (2) topical interests of users are strongly dependent on their level of relationship to their friend's circle. (3) the interest of users is confined to a limited set of topical categories, which can be obtained from their history of POI visits. (4) the choice of POIs are heavily dependent on their popularity.

Problem Statement: Given a set of POIs $V=$

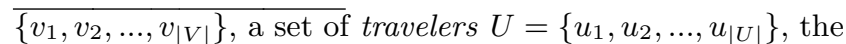
goal of the proposed SSTREC model is to recommend a ranked list of $V$ sequential POIs to a target (or new) traveler $\widetilde{u}$.

\subsection{Generative Process}

The behavior of a traveler is presented as a graphical model (SSTREC) in Figure 3. We describe the generative process of our model as follows:

- A traveler $u$ can decide to visit a venue $v$ based on his own decision or based on the decision of his friends $F_{u}$. This is determined by the distribution of social correlations $\phi^{U F}$ between this user and his friends. Here, $\phi^{U F}$ is a multinomial distribution with symmetric Dirichlet prior, i.e., $\phi^{U F} \sim \operatorname{Dirichlet}(\gamma)$.

- Based on the distribution of social correlations, the user chooses a friend $f_{j, i}$ and selects a POI $i$ by first choosing its category (or topic) $z_{i}$. If the social correlation between the user and his friends is high, $z_{i}$ is drawn from the topical distribution of his friends $\theta_{f}^{U Z}$; if the correlation is low, $z_{i}$ selected from his own distribution $\theta_{u}^{U Z}$.

- The topical preference of the travelers (obtained from their history of POI visits), is incorporated in the form of supervised labels $L$, which acts as a constraint over the topic distribution $\theta^{U Z}$. The label $L^{u}$ of a user $u$ follows a Bernoulli distribution with beta prior, i.e., $L^{u} \sim \operatorname{Bernoulli}(\beta)$.

- A traveler can select a POI in two different ways: (a) based on his (or friend's) topical interest or (b) by simply choosing a POI purely based on it's popularity. In our model,

\footnotetext{
${ }^{1}$ https://developer.foursquare.com/
}

this decision is governed by the variable $d_{i}$ that takes a binary value 0 or 1 .

- If $d$ is 0 :

- The user chooses the venue based on the popularity distribution $\psi^{V} \sim \operatorname{Dirichlet}(\sigma)$.

- If $d$ is 1 :

- The user chooses the venue $v_{i}$ based on (1.) the distribution over the previous POI $v_{i-1}$ and (2.) the topical distribution of POIs, which is a multinomial $\phi^{Z V}$.

Table 1: List of notations used in this paper.

\begin{tabular}{ll}
\hline Symbol & Description \\
\hline $\mathcal{V}=\left\{v_{i}\right\}$ & set of POIs, $v_{i}$ indicates a single POI \\
$U=\left\{u_{i}\right\}$ & set of travelers, $u_{i}$ indicates a single traveler \\
$F=\left\{f_{j}\right\}$ & set of friends of users, where $F \subset U$ \\
$D$ & binary decision variable, representing $d=1$ or $d=0$ \\
$Z=\left\{z_{i}\right\}$ & set of latent topics \\
$K$ & number of topics \\
$L$ & set of observed categories from the history of travelers \\
\hline$\theta^{U Z}$ & topic distribution of travelers \\
$\phi^{U F}$ & distribution of social correlation between $U$ and $F$ \\
$\phi^{Z V}$ & topical distribution of POIs \\
$\lambda$ & $U \times D$ social circle-popularity preference matrix \\
$\psi^{V}$ & popularity distribution of POIs \\
$\alpha, \beta, \gamma$ & hyper-parameters of Dirichlet priors for $\theta^{U Z}, \phi^{U F}$ \\
$\rho, \delta, \sigma$ & hyper-parameters of Dirichlet priors for $\lambda, \phi^{Z V}, \psi^{V}$ \\
\hline$n_{u, f}^{U F}$ & \# times user $u$ preferred friend $f$ 's choice \\
$n_{z, f}^{Z U}$ & \# times a user (or his friend) preferred topic $z$ \\
$n_{z_{i}, v_{i} \mid v_{i-1}}^{Z V}$ & \# times POI $v_{i}$ is assigned to topic $z_{i}$ given $v_{i-1}$ \\
$n_{u, d}^{U D}$ & \# times decision $d$ is picked by user $u$ \\
$n_{v}^{V}$ & \# times POI $v$ is picked only based on popularity \\
\hline
\end{tabular}

\section{PARAMETER ESTIMATION}

We adopt a collapsed Gibbs sampling for posterior inference of SSTREC parameters $\left(\phi^{U F}, \theta^{U Z}, \lambda, \phi^{Z V}\right.$ and $\left.\psi^{V}\right)$. The posterior probability of our model is:

$$
p(z, f, d \mid v, \beta, \alpha, \sigma, \delta, \rho, \lambda)=\frac{p(z, f, d, v \mid .)}{p(v \mid .)}
$$

Direct multinomial relationship between the variables $f$ and $z$ creates complex inter-dependencies of two latent variables. To overcome this problem, we first estimate the social correlations $\phi^{U F}$ by using the traditional LDA topic model [2], where the observed words are the set of friends in our data, and the documents are the users. After this step, we treat $\phi^{U F}$ as an observed variable (denoted by the shaded circle in Figure 3).

Using the generative process, the total likelihood can be expanded as follows:

$$
\begin{aligned}
& p(f, z, d, v \mid .) \\
& =\int p\left(f \mid \phi^{U F}\right) p\left(\phi^{U F} \mid \gamma\right) d \phi^{U F} \cdot \int p(d \mid \lambda) p(\lambda \mid \rho) d \lambda \\
& \cdot \int p\left(z \mid f, \theta^{U Z}\right) p\left(\theta^{U Z} \mid \alpha, \mathcal{L}\right) d \theta^{U Z} \\
& \cdot \iint p\left(v_{i} \mid v_{i-1}, d, z, \psi^{V}, \phi^{Z V}\right) p\left(\psi^{V} \mid \sigma\right) p\left(\phi^{Z V} \mid \delta\right) d \psi^{V} \phi^{Z V}
\end{aligned}
$$




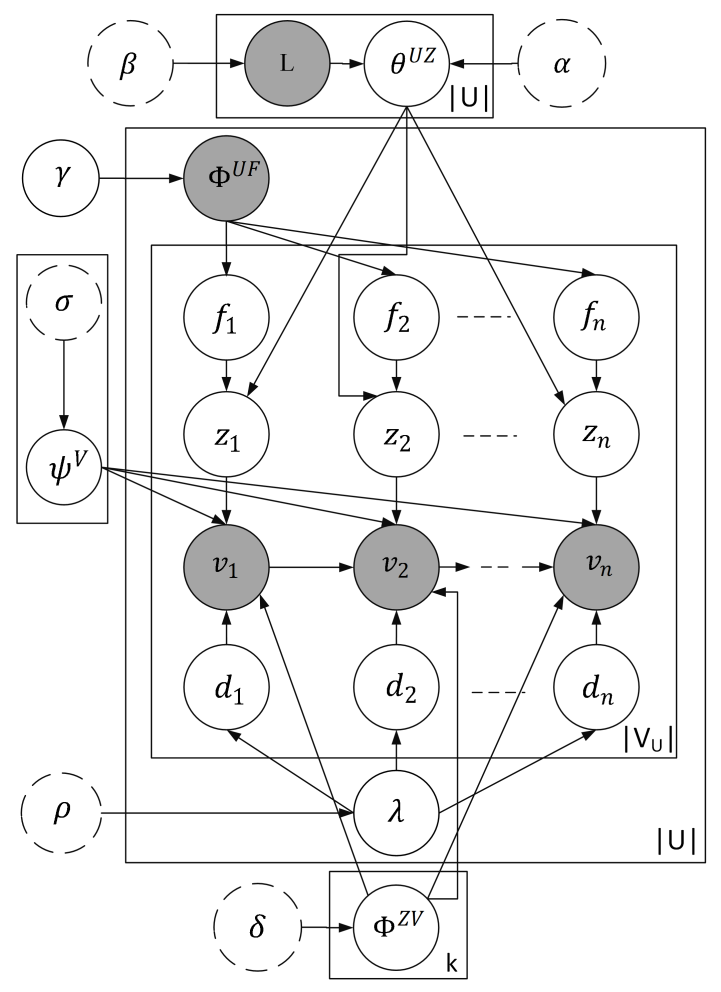

Figure 3: Plate diagram for generative process of SSTREC.

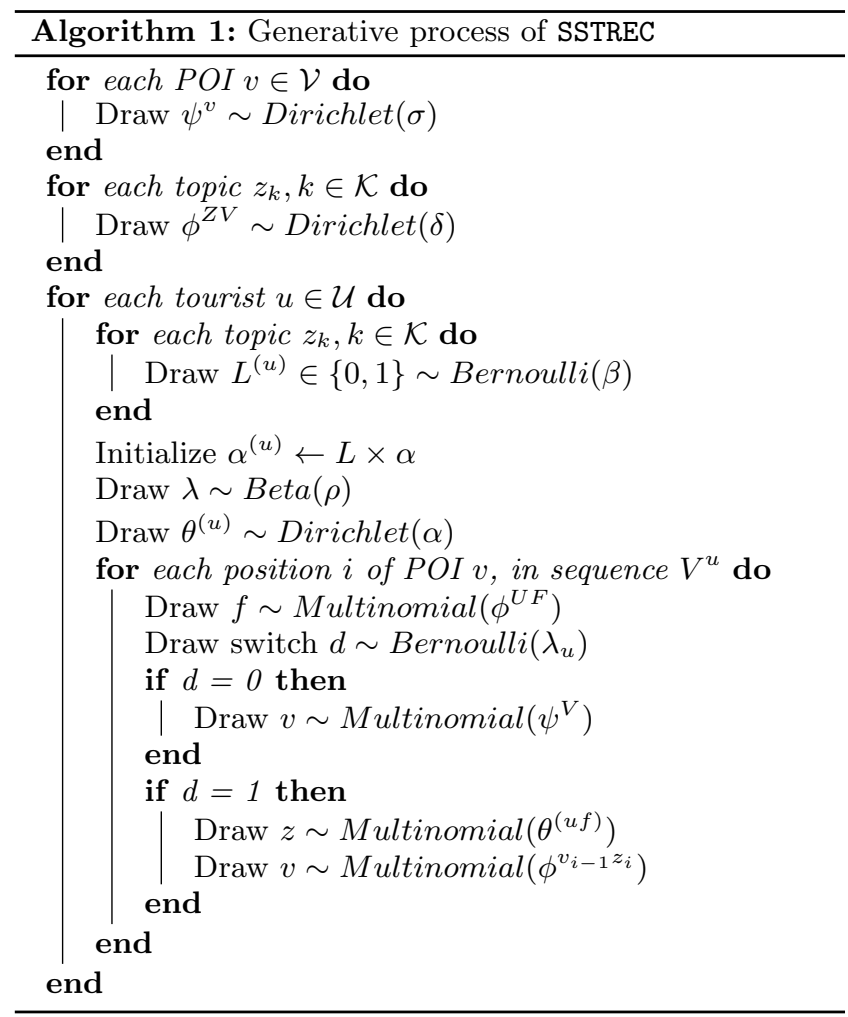

According to Algorithm 1, the selection of POI by a user is based on two distinct choices, namely, popularity and personal choice. This allows to decompose the last multiplier in Equation (2) into a product of two independent components: $\underbrace{\int p\left(v_{i}^{(0)} \mid d, \psi^{V}\right) p\left(\psi^{V} \mid \sigma\right) d \sigma}_{\text {popularity }} \cdot \underbrace{\int p\left(v_{i}^{(1)} \mid v_{i-1}, d, z, \phi^{Z V}\right) p\left(\phi^{Z V} \mid \delta\right) d \delta}_{\text {personal choice }}$

In the above equation, $v_{i}^{0}$ indicates the POI that is selected when $d=0$ and $v_{i}^{1}$ corresponds to the POI chosen when $d=1$. There are two important notes about Equation 2: first, the labeling prior $\beta$ does not have a direct impact over the model; hence, it is not included in the equation. This is because, once the labels $\mathcal{L}$ are observed, $\beta$ becomes d-separated [24]. Second, since we infer the $\phi^{U F}$ using an independent LDA model, the distribution of friendship correlation is now observed, which makes the $\gamma$ d-separated as well.

Since $f$ is a multinomial that directly affects $z$, we first sample $z$ for all combinations of $f$. Based on Equations (2) and (3) the posterior is given by:

$$
\begin{aligned}
& p\left(z_{i}=k, f_{i}=f \mid v^{(1)}, z_{\neg i}, f_{\neg i}\right) \\
& =\frac{\int p\left(f \mid \phi^{U F}\right) p\left(\phi^{U F} \mid \gamma\right) d \gamma}{\int p\left(f_{\neg j} \mid \phi^{U F}\right) p\left(\phi^{U F} \mid \gamma\right) d \gamma} \cdot \frac{\int p\left(z \mid f, \theta^{U Z}\right) p\left(\theta^{U Z} \mid \alpha\right) d \theta^{U Z}}{\int p\left(z_{\neg i} \mid f, \theta^{U Z}\right) p\left(\theta^{U Z} \mid \alpha\right) d \theta^{U Z}} \\
& \cdot \frac{\int p\left(v_{i}^{1} \mid v_{i-1}, d, z, \phi^{Z V}\right) p\left(\phi^{Z V} \mid \beta\right) d \phi^{Z V}}{\int p\left(v_{i}^{1} \mid v_{i-1}, d, z_{\neg i}, \phi^{Z V}\right) p\left(\phi^{Z V} \mid \beta\right) d \phi^{Z V}} \\
& \propto \frac{n_{u, f, \neg i}^{U F}+\gamma_{f u}}{\sum_{f^{\prime}}^{F}\left(n_{u, f^{\prime}, \neg i}^{U F}+\gamma_{f^{\prime} u}\right)} \cdot \frac{n_{z, f, \neg i}^{Z F}+\alpha_{z}}{\sum_{z=k}^{K}\left(n_{z, f, \neg i}^{Z F}+\alpha_{z}\right)-1} \cdot \frac{n_{z_{i}, v_{i} \mid v_{i-1}, \neg i}^{Z V}+\delta_{v_{i} \mid v_{i-1}}}{\sum_{v^{\prime}}^{V}\left(n_{z_{i}, v^{\prime}, \neg i}^{Z V}+\delta_{v^{\prime}}\right)-1}
\end{aligned}
$$

Once we sample the topics $z$ for all combinations of friends, we then sample for the decision variable $d=1$ and $d=0$ as follows:

$$
\begin{aligned}
& p\left(d_{i}=1 \mid d_{\neg i}, z, v, f\right)= \\
& \frac{\int p(d \mid \lambda) p(\lambda \mid \rho) d \lambda}{\int p\left(d_{\neg i}\right) p(\lambda \mid \rho) d \lambda} \cdot \frac{\int p\left(v_{i}^{(1)} \mid v_{i-1}, d, z, \phi^{Z V}\right) p\left(\phi^{Z V} \mid \delta\right) d \phi^{Z V}}{\int p\left(v_{i}^{(1)} \mid v_{i-1}, d_{\neg i}, z, \phi^{Z V}\right) p\left(\phi^{Z V} \mid \delta\right) d \phi^{Z V}} \\
& \propto \frac{n_{u,(1), \neg i}^{U D}+\rho}{n_{u,(1), \neg i}^{U D}+n_{u,(0), \neg i}^{U D}+2 \rho-1} \cdot \frac{n_{z_{i}, v_{i} \mid v_{i-1}, \neg i}^{Z V}+\delta_{v}}{\sum_{v^{\prime}}^{V}\left(n_{z_{i}, v^{\prime}, \neg i}^{Z V}+\delta_{v^{\prime}}\right)-1} \\
& \quad p\left(d_{i}=0 \mid d_{\neg i}, z, v, f\right) \\
& \propto \frac{n_{u,(0), \neg i}^{U D}+\rho}{n_{u,(0), \neg i}^{U D}+n_{u,(1), \neg i}^{U D}+2 \rho-1} \cdot \frac{n_{v, \neg i}^{V}+\sigma_{v}}{\sum_{v^{\prime}}^{V}\left(n_{v^{\prime}, \neg i}^{V}+\sigma_{v^{\prime}}\right)-1}
\end{aligned}
$$

Once the topics $z$ and decision variables $d$ are sampled, the estimated parameters of the model $\hat{\theta}^{U Z}, \hat{\lambda}, \hat{\phi}^{Z V}$, and $\hat{\psi}^{V}$ can be derived by normalizing the counts $n^{N F}, n^{U D}, n^{Z V}$, and $n^{V}$, respectively.

Recommending POIs: Given a traveler $\widetilde{u}$, and his current venue $v_{i-1}$ the recommendation score of an unseen (or next) POI $\widetilde{v}_{i}$ is calculated as:

$$
p\left({\widetilde{v^{\prime}}}^{\prime} \mid v_{i-1}, \widetilde{u}\right)=\sum_{z \in K} \hat{\theta}_{\widetilde{u}, z}^{U Z} \cdot \hat{\phi}_{z, \widetilde{v}^{\prime} i \mid v_{i-1}}^{Z V} \cdot \hat{\lambda}_{\widetilde{u}}+\left(1-\hat{\lambda}_{\widetilde{u}}\right) \cdot \hat{\psi}_{v}^{V}
$$

One can observe that Equation (7) supports the set of all behavioral traits of travelers that were outlined in Section 2. First, parameter $\theta^{\hat{U} Z}$ captures the favorite categories of travelers, $\phi^{\hat{Z} V}{ }_{v_{i} \mid v_{i-1}}$ captures the sequential relationship 
between the POIs, and $\hat{\psi}^{V}$ captures the popularity-based POI preference. It is important to note that the distribution $\theta^{\hat{U} Z}$ also encompasses the social component of our model, since according to our generative model, the topical space of users is constrained by the selection of friends.

\subsection{Incorporating Prior Information}

Our proposed model is a semi-supervised generative framework, which allows us to incorporate traveler and POIspecific features as priors.

Prior Information for Travelers: the traveler-based features are two-fold: (a) POI categories from the historical visits of users, which form the supervised topical labels $\mathcal{L}$ and (b) strength of friendship, which correspond to the prior information for the distribution $\phi^{U F}$. The prior $\beta$ for the label $\mathcal{L}$ is defined as follows:

$$
\beta(u, c)=\frac{\# \text { times user } u \text { visits POI with category } c}{\text { total POI visits by } u}
$$

The priors for social relationship $\gamma$ is determined using three different features, namely (1) the presence of a friendship link between users, (2) the number of overlapping topical categories (obtained from historical POI visits) and (3) the number of lists that are commonly followed by the users. In Foursquare, lists are like folders which enable the users to organize POIs that share similar characteristics in the form of geographical proximity or topical categories. This feature is extremely useful since it expresses the explicit action of users' interest over a collection of POIs. Consequently, the strength of friendship between users $a$ and $b$ is formulated as a linear combination of the above features as follows:

$$
\gamma(a, b)=I(a, b) w+C a t(a, b)+\ell(a, b)
$$

In the above equation, $w$ is a weight factor, $I$ is the indicator variable which denotes the presence of link between users $a$ and $b, C a t$ and $\ell$ denote the categorical and lists features respectively.

Prior Information for POIs: Popularity based features are introduced as prior $\sigma$ into our model. We formulate the popularity score of a POI $v$ as a function of the two features namely, the number of prominent reviewers for a POI $\mathcal{R}$ (explained in Section 2) and the total number of check-ins $\mathcal{M}$. Formally, this prior is calculated as $\sigma(v)=\mathcal{R}(v)+$ $\mathcal{M}(v)$.

\subsection{Creating POI Sequences}

The ultimate goal of the proposed method is to recommend a series of POIs that can serve as travel itineraries to the users. Inspired by the algorithm proposed in [14], we assume that a traveler will provide the following inputs to our model: (1) the current location, (2) the arrival time, (3) the number of route options, and (4) the spare time, which indicates the total time a traveler is willing to spend during his current trip. The procedure for route generation is shown in Algorithm 2, where the spare time is indicated by $B, b$ denotes buffer time, and $K$ denotes the number of route options. The algorithm starts by inserting the start location of the user $v_{u}^{\text {start }}$ to the priority queue $Q$ as the very first sequence (a single POI is a special case of a sequence). It then generates $K$ travel routes by performing the following set of operations. First, it pops the sequence with highest weight (i.e. the first POI $v_{u}^{\text {start }}$ in this case) from the priority queue and checks if it meets our distance criteria (line $5)$. This distance $d^{r}$ should be greater than the total travel time, which is the spare time plus the buffer time. If yes, then the algorithm acknowledges this as a route for recommendation (line 6); if not, it looks for alternative sequences of routes in lines 9-15, where it calculates the posterior according to the SSTREC model and the total distance for each new route in lines 11 and 12 and adds this new route along with other metadata to the priority queue. In this algorithm, $r v+$ denotes a new POI $v$ being added to route $r, r\left[v_{l}\right]$ is the last visited venue in the route, $p^{r v+}$ and $d^{r v+}$ indicates the updated probability and distance for the sequence $r v+$ respectively. To reduce the number of POI combinations in our Gibbs sampling algorithm, we only consider POIs that meet the distance threshold.

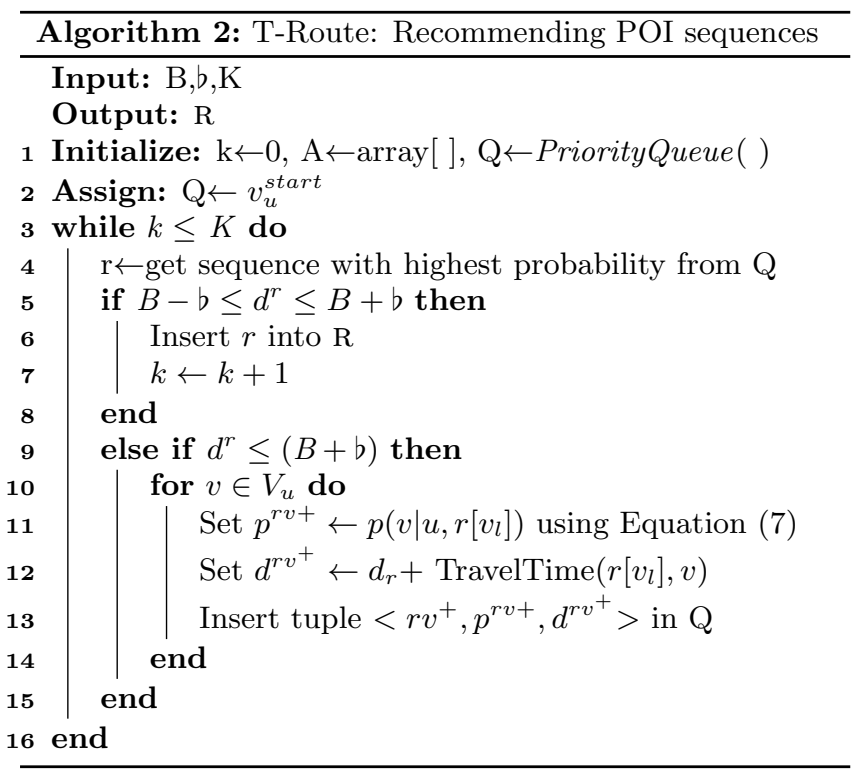

\section{EXPERIMENTS}

In this section, we report the results of comprehensive experimental evaluation of the proposed SSTREC model and compare it with other baselines and the state-of-the-art probabilistic model. We begin by discussing the details of our data collection methodology, which is then followed by the explanation of the evaluation metrics and the results of our experiments.

\subsection{Dataset Description}

For our experiments, we obtained the tweets of foursquare check-ins from the authors of [15], which spans from MarchJuly 2014. We then augmented this raw data with a variety of POI and user-profile information by querying the Foursquare API. Information about POI includes textual description, rating, etc. Information about user includes friends, number of check-ins, and lists. In total, we obtained $1,247,847$ check-ins by 108,341 unique users and 170,472 venues (POIs) distributed over 20 cities. In this paper, we perform our experiments over a subset of this data, which pertains to the top four cities based on the frequency of check-ins. The statistics of our dataset is shown in Table 2, where $M n$ denotes mean, $M d$ the median, $C h k$ indicates check-ins, Frns denotes friends and Cats corresponds to the categories (or topics) of POIs. Using this dataset as the base, 
we mimic a real-world scenario of travelers by taking every location, and creating three new cases based on the following conditions: (1) Tourist dataset (D1): Every user should have checked-in for at least 2 consecutive days and at most 6 days. The home location of the user should be different from the target city. For instance, if we are recommending POIs in Chicago, the user location should not be from Chicago. (2) Localè dataset (D2): We relax the constraint on consecutive check-ins; meaning, we do not care about whether a person has checked-in on consecutive days. However, for this case, the home location of the user should be the same as the target location. (3) Social dataset (D3): We remove constraints that was set for D1 and D2. Nonetheless, for this case, every user should have atleast 4 friends (social connection) with every member who is a part of this dataset. It should be noted that the statistics shown in Table 2 vary for each of the above cases (i.e. D1-D3). Researchers can download the raw datasets used in our experiments from our public Github repository ${ }^{2}$.

Table 2: Statistics of our Foursquare dataset.

\begin{tabular}{|l|c|c|c|c|c|c|}
\hline City & \#Users & \#POI & $\begin{array}{c}\text { Mn } \\
\text { Chk }\end{array}$ & $\begin{array}{c}\text { Md } \\
\text { Chk }\end{array}$ & $\begin{array}{c}\text { Mn } \\
\text { Frns }\end{array}$ & $\begin{array}{c}\text { Mn } \\
\text { Cats }\end{array}$ \\
\hline NY & 1521 & 2076 & 10.75 & 8 & 10.04 & 17.14 \\
\hline Moscow & 1132 & 1574 & 13.05 & 7 & 5.80 & 9.36 \\
\hline LA & 794 & 976 & 8.51 & 6 & 9.37 & 13.45 \\
\hline Chicago & 822 & 1079 & 8.9 & 6 & 7.4 & 13.36 \\
\hline
\end{tabular}

\subsection{Performance Evaluation}

The evaluation methodology for our model consists of two types; the first type is called the uni-step recommendation and the second type is termed as the multi-step recommendation. In the uni-step evaluation, we use the first $n-1$ visited POIs in the sequence for training and the last visited POI for testing. In multi-step evaluation, instead of removing only the last visited POI, we use the last 3 contiguous POI sequences for testing. We make sure that the testing POIs fall within the spare time plus the buffer time that was mentioned in Section 4.2. Our evaluation is performed over all cases of the dataset, namely, Tourist (D1), Locale (D2) and Social (D3). The SSTREC model and all other baselines are implemented using Python's numpy numerical module, and Scikit-Learn machine learning module ${ }^{3}$.

\subsubsection{Evaluation Metrics}

To evaluate the performance of ranking, we use the standard information retrieval measures. For every traveler, we compute: (1) $\mathrm{P} @ \mathrm{~N}$ : precision at rank $N$ is the fraction of POIs that were actually visited by users in the top- $N$ ranked POI instances, (2) $\mathrm{R} @ \mathrm{~N}$ : recall at rank $N$ is the fraction of the visited POIs that were retrieved at every top- $N$ ranked POI instances, (3) S@N: The success at rank $N$ is the probability of finding at least one truly visited POI in the top$N$ ranked set, (4) DCG: The discounted cumulative gain is based on the fact that highly relevant POIs are more important than marginally relevant ones and (5) Edit distance: The minimum number of operations that are required to transform the recommended sequence of POIs to the se-

\footnotetext{
${ }^{2}$ https://github.com/magnetpest2k5/WSDM17

${ }^{3}$ http://scikit-learn.org
}

quence of POIs in the actual ground-truth (i.e., test set) of the user.

\subsubsection{Baseline Methods for Comparison}

We compare the performance of our proposed model with the following baselines:

- Random Multinomial Choice $(R C)$ recommends a location by naively drawing a location $v$ from a multinomial distribution of global location weights $v_{i} \sim$ $\operatorname{Multi}\left\{v_{1}, \ldots, v_{n}\right\}$. These weights are not user-specific and simply based on the number of check-ins for each location.

- Markov Model ( $M M)$ predicts the next visiting venue of the user using a Markov model that calculates the probability $p\left(v_{t} \mid v_{t-1}\right)$, where $v_{t}$ is the next (to be visited) landmark, $v_{t-1}$ is the previously visited landmark. This model completely ignores the topical interests of users.

- Photographer Behavior Model (PBM) is a state-of-theart topic model that uses a combination of Markov model and PLSA topic model [22] to recommend sequence of POIs [14].

\subsection{Experimental Results}

\subsubsection{Recommending Singular POI Sequence}

We begin this section, by presenting the performance of the model in terms of recall in Figure 4. In general, the proposed SSTREC model outperforms other models on all dataset types, while the random multinomial choice (RC) has the worst performance. Although PBM performs better than the Markov model (MM), it is important to note that the results are not consistent. For instance, in Figure 4(b), the performance of PBM is lower than MM on D1 and D2 datasets. On the other hand, the results of the proposed model are consistent throughout all the scenarios. A possible explanation for such inconsistency is that PBM relies on a naive combination of Markovian and the topic probability; this makes the topic space disjoint from the POI transition probability. Contrary to this, SSTREC learns the preference of POIs using a unified generative framework where the topic space of travelers and POIs are constrained on Markovian transition probabilities, popularity of POIs and categorical choice of users. The best performance of SSTREC (and all other models) is observed over the social dataset D3. This is mainly due to the nature of this dataset, where the presence of friendship links between the users results in many commonly visited POIs. Additionally, our model is able to leverage this social linkage to overcome sparsity and yield better results. The precision performance of the models shown in Figures 4(e)-(h) are similar to their recall counterparts with SSTREC outperforming other models by achieving a precision up to $10 \%$. The PBM closely follows our model, but not for all scenarios; its poor consistency is yet again revealed in Figures 4(f) and 4(g), where MM outperforms all other models except SSTREC.

Quality of Ranked POIs: DCG is a classic performance measure that is used widely in evaluating information retrieval systems. In our setting, we use this measure to penalize incorrectly ranked POIs based on their positions. Unlike information retrieval, where documents are assigned different relevance levels, our data is binary (i.e. 1 if user visits a POI and 0 otherwise); consequently, we set a constant relevance score of 3 for all POIs. The comparison of 


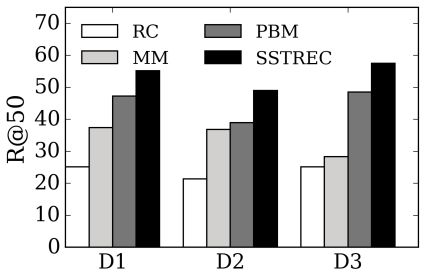

(a) New York

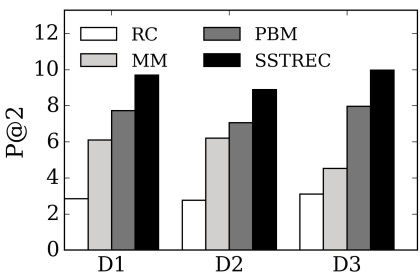

(e) New York

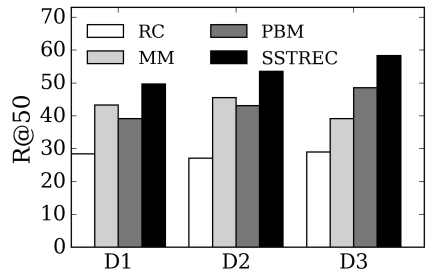

(b) Moscow

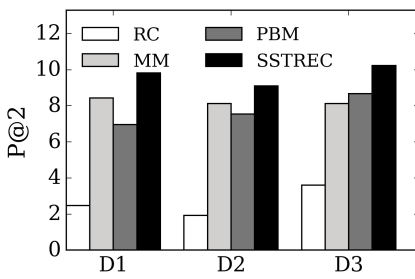

(f) Moscow

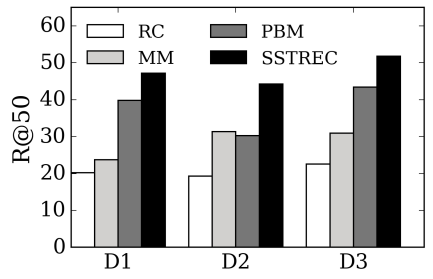

(c) Chicago

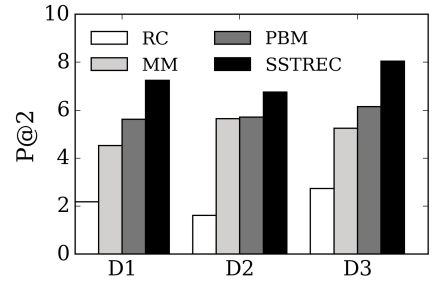

(g) Chicago

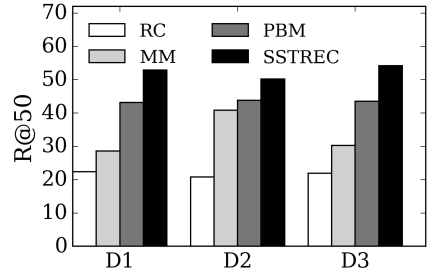

(d) Los Angeles

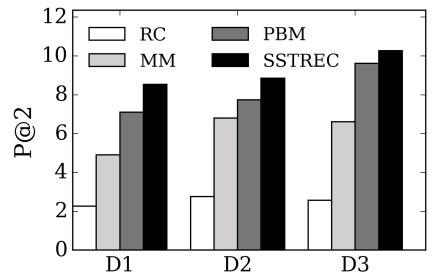

(h) Los Angeles

Figure 4: Performance comparison of SSTREC model: (a)-(d) shows the recall scores and (e)-(h) show the precision scores.

DCG scores between SSTREC and PBM for top 50 ranked POIs is shown in Figures 5(a)-(d), where the x-axis denotes the topic size. We see that SSTREC performs better than PBM over both the data set types D1 and D3. It should be noted that SSTREC is a supervised model where the topic space corresponds to the number of unique POI categories. Therefore, the topic count for this model corresponds to the number of unique POI categories. The DCG scores of PBM reaches a saturation point at about 20 topics. Contrary to this, SSTREC performs better with more topics since this essentially translates into more supervised information. In our experiments, we were able to see a steady improvement in DCG even beyond 100 topics, although the improvement was marginal. Originally, we have 320 unique categories of POIs from Foursquare.

\subsubsection{Recommending Multiple POI Sequences}

In this experiment, we focus on evaluating the exact order of POI sequences generated by Algorithm 2; i.e., a recommended sequence is deemed as a true positive instance, only if it matches with the exact order of the POIs sequentially visited by a user in the test data. The outcome of this experiment is depicted as edit distance in Figure 5, which is based on the following edit operations: insert into a sequence, delete from a sequence, and replace one POI with another. From the results, we observe that the proposed SSTREC model has the lowest edit distance among all models. As the spare time increases, the number of recommended venues increases as well, which in-turn increases the number of mismatches in the recommended sequences. Unlike the precision and recall scores, we did not find any major performance increase between datasets D1 and D3.

\subsubsection{Visual Interface for Travelers}

In this section, we present the qualitative results of our model using a visual interface shown in Figure 6. Due to space limitations, we restrict our example to just one user and one city. In this interface, the user provides the input city as New York, a spare time of 4 hours and the start location as Union square (denoted by red star) and the number of route options as 3. Based on the topical interest of the user (not shown in the Figure, since it is background information) and the travel time between POIs, the interface shows 3 recommended sequences of POIs with varying travel times. The topics associated with the sequences are presented above them. For instance, route 1 consists of Chelsea market, 9/11 Memorial, Rockwood Music Hall that are associated with Historical sites, Music and Shopping. It can be seen that all routes that are recommended have historical site and music as common topical categories. Although there is some mismatch between topics and POI sequences, for most part they are coherent. In addition to the recommended route sequences, the figure also shows the topics and POIs from the user's social network, which is provided by leveraging the distribution of social correlations. It should be noted that the travel times indicated on the routes are not the exact travel times; instead, they correspond to a combination of travel and visiting time. For example, the travel time of 1.5 hours between POI 5 and 6 indicates the time taken to reach POI 6 from 5, plus the time to tour POI 6.

\section{RELATED WORK}

Research on location recommendation can be broadly classified into the following three categories: (a) simple POI recommendation that aims at suggesting individual and independent landmarks, (b) travel package recommendation and (c) tour recommendation.

Recommending Independent POIs: There are numerous works on recommending independent venues. Matrix factorization techniques to recommend POIs in LSBNs are proposed in $[6,1,38]$, while [6] incorporates temporal properties into these models. In [32], the authors propose a power-law probabilistic model, [3] formulates the probability of a user's check-in as a Multi-center Gaussian model, and [21] integrates user preference and location into a Bayesian learning model. The authors of [18] and [33] incorporate contextual information into a topic modeling based framework, while [29] proposed a hybrid matrix factorization model to incorporate sentiments. A more recent work on location recommendation addresses the cold start problem by viewing non-visited locations as non-negative samples and proposes a content-aware collaborative filtering [16]. Lian et 


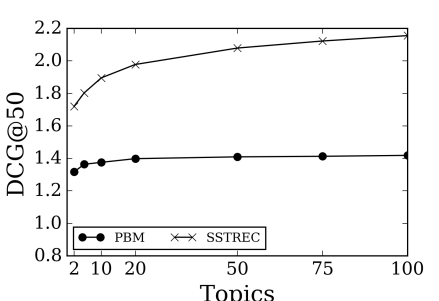

(a) Los Angeles D1

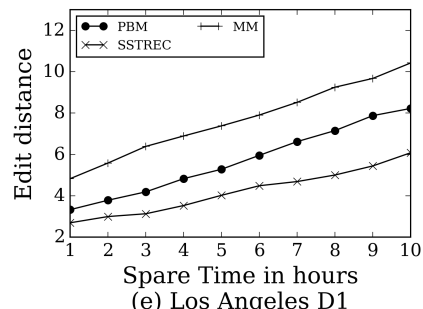

(e) Los Angeles D1

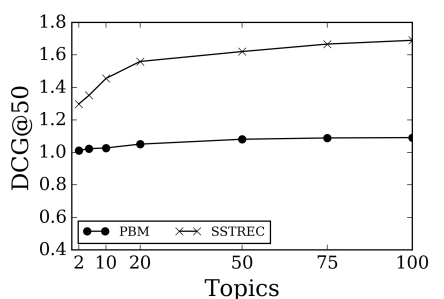

(b) New York D1

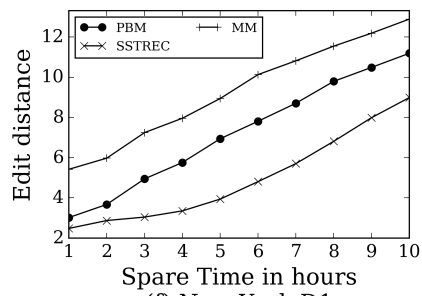

(f) New York D1

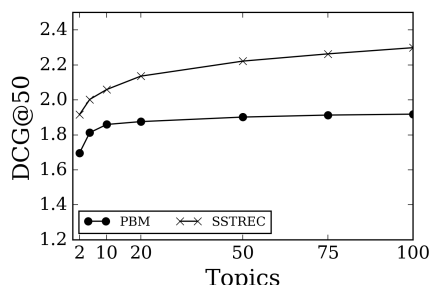

(c) Los Angeles D3

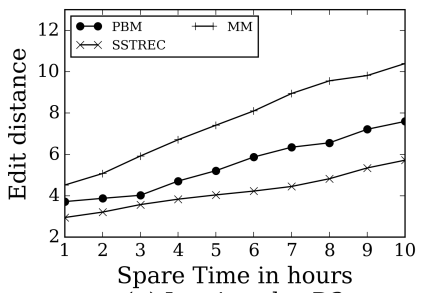

(g) Los Angeles D3

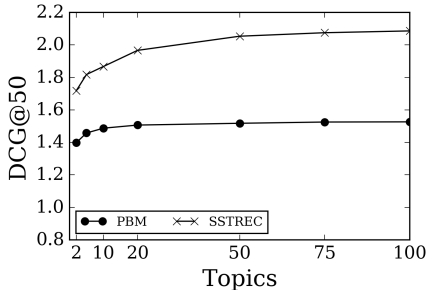

(d) New York D3

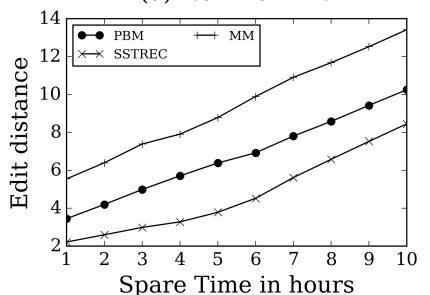

(h) New York D3

Figure 5: (a)-(d) shows the performance comparison of SSTREC model in terms of DCG. (e)-(f) shows the variation in the recommended POI sequences for travelers.

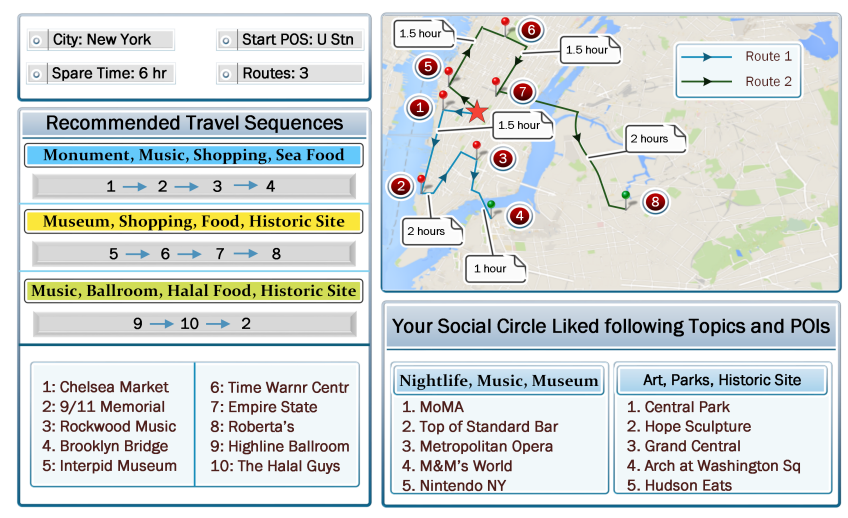

Figure 6: A visual example of travel routes recommended by the SSTREC model.

al. [17] addresses the same problem by viewing mobility records as implicit feedback and leverages them as weighted matrix factorization. The authors of [34, 10, 15] adopt a different methodology of suggesting location by segmenting geographical areas into sub-regions based on the characteristics of POIs. Topic models incorporating geographical and social information have also been shown to be effective for other tasks, such as opinion mining [30] and social media information retrieval $[11,13,12]$.

Travel package recommendation: utilizes the geo-location information of travelers to recommend vacation packages such as a combined package of rental car plus hotel stay or flight travel hotels and local transportation [19, 7]. Nonetheless, this body of work is different from ours, since their goal is focused on creating combination of attractive packages that might draw the attention of travelers. On the other hand, our goal is to recommend a combination (more specifically a sequence) of points of interest for travelers. Although [20] and [4] consider the sequential pattern of POI visits, they do not factor in other important features, such as popularity of locations and social networks of users. Additionally, these methods recommend only single POI rather than their sequence.
Travel Route Recommendation: Unlike the above mentioned body of works, travel route recommendation is an emerging area, where most published papers are relatively new $[25,35,36,37,27]$. In [36], the authors adopt a collaborative retrieval model that incorporates pairwise weighted approximate rank function, while [37] proposes a pairwise tensor factorization-based framework that models user-POI, POI-time, and POI-POI interactions for successive POI recommendation. The authors of [39] model the interests of travelers using the popular HITS algorithm. By utilizing GPS logs from mobile devices various travel sequences are suggested for the users. Goinis et al. [8] adopted a timeaware tour recommendation framework that optimizes travel routes based on the best visiting times of POIs and [9] proposed algorithms that incorporate various constraints, such as variety of venues, budget constraints of users and the satisfaction provided by the POIs for recommendation. In a recent work, Wen et al. [28] incorporate the semantics of keywords from user queries in a skyline travel route framework for creating sequential POIs.

Despite being novel, the recommendation frameworks proposed in these works provide a very low degree of personalization. Some consider time-dependent factors, but ignore the topical preference of users; some capture user-level features, but do not incorporate temporal or sequential visiting patterns. In summary, our paper is uniquely different from the above mentioned works because of the following reasons: (1) we propose a novel topic-model based approach that incorporates the temporal quality of sequential visits, influence from social network of the user, the topical preference of users and the popularity of POIs. (2) using the proposed generative model, we recommend a series of travel sequences that will interest tourists. The paper that is closest to our work [14] uses a combination of a topic model and a Markov model to recommend sequences of venues. Therefore, in this paper, we treat this model as the state-of-the-art tour recommendation system and compare it with the proposed SSTREC recommendation framework. 


\section{CONCLUSION}

In this paper, we developed a social sequential tour recommendation SSTREC model, which provides personalized POI recommendations for travelers. Using a novel generative approach, the proposed model utilizes diverse features, such as temporal sequences, social relations, topical preferences and popularity of POIs, to provide quality POI recommendations to travelers. The model was then extended using a best first search algorithm to recommend a sequence of POIs that could serve as travel itineraries. Using extensive set of experiments, and a rich dataset of Foursquare check-ins, we showed that our model outperforms a state-of-the-art probabilistic model in almost all scenarios.

\section{Acknowledgements}

This work was supported in part by the National Science Foundation grants IIS-1707498, IIS-1619028 and IIS1646881 .

\section{REFERENCES}

[1] Berjani, B., And Strufe, T. A recommendation system for spots in location-based online social networks. In Proceedings of the 4 th Workshop on Social Network Systems (2011), ACM.

[2] Blei, D. M., NG, A. Y., AND Jordan, M. I. Latent dirichlet allocation. Journal of machine Learning research 3, Jan (2003), 993-1022.

[3] Cheng, C., Yang, H., King, I., And Lyu, M. R. Fused matrix factorization with geographical and social influence in location-based social networks. In Twenty-Sixth AAAI Conference on Artificial Intelligence (2012).

[4] Cheng, C., Yang, H., Lyu, M. R., And King, I. Where you like to go next: successive point-of-interest recommendation. In Proceedings of the Twenty-Third International Joint Conference on Artificial Intelligence (2013), AAAI Press, pp. 2605-2611.

[5] Cheng, Z., Caverlee, J., Lee, K., and Sui, D. Z. Exploring millions of footprints in location sharing services. ICWSM 2011 (2011), 81-88.

[6] Gao, H., Tang, J., Hu, X., and LiU, H. Exploring temporal effects for location recommendation on location-based social networks. In Proceedings of the 7th ACM conference on Recommender systems (2013), ACM, pp. 93-100.

[7] Ge, Y., Liu, Q., Xiong, H., Tuzhilin, A., And Chen, J. Cost-aware travel tour recommendation. In Proceedings of the 17th ACM SIGKDD International Conference on Knowledge Discovery and Data mining (2011), ACM, pp. 983-991.

[8] Gionis, A., Lappas, T., Pelechrinis, K., and Terzi, E. Customized tour recommendations in urban areas. In Proceedings of the 7th ACM international conference on Web search and data mining (2014), ACM, pp. 313-322.

[9] HsieH, H.-P., AND Li, C.-T. Mining and planning time-aware routes from check-in data. In Proceedings of the 23rd ACM International Conference on Conference on Information and Knowledge Management (2014), ACM, pp. 481-490.

[10] Hu, B., And Ester, M. Spatial topic modeling in online social media for location recommendation. In Proceedings of the 7th ACM conference on Recommender systems (2013), ACM, pp. 25-32.

[11] Kotov, A., And Agichtein, E. The importance of being socially-savvy: quantifying the influence of social networks on microblog retrieval. In Proceedings of the 22nd ACM International Conference on Information $\&$ Knowledge Management (2013), ACM, pp. 1905-1908.
[12] Kotov, A., Rakesh, V., Agichtein, E., And Reddy, C. K. Geographical latent variable models for microblog retrieval. In Proceedings of the 37th European Conference on Information Retrieval (2015), ACM, pp. 635-647.

[13] Kotov, A., Wang, Y., And Agichtein, E. Leveraging geographical metadata to improve search over social media. In Proceedings of the 22nd International Conference on World Wide Web (2013), ACM, pp. 151-152.

[14] Kurashima, T., Iwata, T., Irie, G., And Fujimura, K. Travel route recommendation using geotags in photo sharing sites. In Proceedings of the 19th ACM International Conference on Information and Knowledge Management (2010), ACM, pp. 579-588.

[15] Le Falher, G., Gionis, A., and Mathioudakis, M. Where is the soho of rome? measures and algorithms for finding similar neighborhoods in cities. In $A A A I$ Conference on Web and Social Media (2015).

[16] Lian, D., Ge, Y., Zhang, F., Yuan, N. J., Xie, X., Zhou, T., AND RuI, Y. Content-aware collaborative filtering for location recommendation based on human mobility data. In Data Mining (ICDM), 2015 IEEE International Conference on (2015), IEEE, pp. 261-270.

[17] Lian, D., Zhao, C., Xie, X., Sun, G., Chen, E., and Rui, Y. Geomf: Joint geographical modeling and matrix factorization for point-of-interest recommendation. In Proceedings of the 20th ACM SIGKDD International Conference on Knowledge Discovery and Data mining (2014), ACM, pp. 831-840.

[18] LiU, B., AND Xiong, H. Point-of-interest recommendation in location based social networks with topic and location awareness. In SDM (2013), vol. 13, SIAM, pp. 396-404.

[19] Liu, Q., Ge, Y., Li, Z., Chen, E., And Xiong, H. Personalized travel package recommendation. In Data Mining (ICDM), 2011 IEEE 11th International Conference on (2011), IEEE, pp. 407-416.

[20] Liu, X., Liu, Y., Aberer, K., And Miao, C. Personalized point-of-interest recommendation by mining users' preference transition. In Proceedings of the 22nd $A C M$ International Conference on Information $\&$ Knowledge Management (2013), ACM, pp. 733-738.

[21] Park, M.-H., Hong, J.-H., And Cho, S.-B. Location-based recommendation system using bayesian user's preference model in mobile devices. In Ubiquitous Intelligence and Computing. Springer, 2007, pp. 1130-1139.

[22] Popescul, A., Pennock, D. M., and Lawrence, S. Probabilistic models for unified collaborative and content-based recommendation in sparse-data environments. In Proceedings of the Seventeenth conference on Uncertainty in artificial intelligence (2001), Morgan Kaufmann Publishers Inc., pp. 437-444.

[23] Rakesh, V., Lee, W.-C., And Reddy, C. K. Probabilistic group recommendation model for crowdfunding domains. In Proceedings of the Ninth ACM International Conference on Web Search and Data Mining (2016), ACM, pp. $257-266$.

[24] Ramage, D., Hall, D., Nallapati, R., and Manning, C. D. Labeled lda: A supervised topic model for credit attribution in multi-labeled corpora. In Proceedings of the 2009 Conference on Empirical Methods in Natural Language Processing: Volume 1- (2009), Association for Computational Linguistics, pp. 248-256.

[25] Roy, S. B., Das, G., Amer-Yahia, S., and Yu, C. Interactive itinerary planning. In IEEE 27th International Conference on Data Engineering (2011), IEEE, pp. 15-26.

[26] Wallach, H. M. Topic modeling: beyond bag-of-words. In Proceedings of the 23rd International Conference on Machine Learning (2006), ACM, pp. 977-984.

[27] Wang, W., Yin, H., Sadiq, S., Chen, L., Xie, M., And ZHou, X. Spore: A sequential personalized spatial item recommender system. In IEEE 32nd International Conference on Data Engineering, ICDE, (2016). 
[28] Wen, Y.-T., Cho, K.-J., Peng, W.-C., Yeo, J., And HwANG, S.-W. Kstr: Keyword-aware skyline travel route recommendation. In Data Mining (ICDM), IEEE International Conference on (2015), IEEE, pp. 449-458.

[29] Yang, D., Zhang, D., Yu, Z., And Wang, Z. A sentiment-enhanced personalized location recommendation system. In Proceedings of the 24th ACM Conference on Hypertext and Social Media (2013), ACM, pp. 119-128.

[30] Yang, Z., Kotov, A., Mohan, A., And Lu, S. Parametric and non-parametric user-aware sentiment topic models. In Proceedings of the 38th International ACM SIGIR Conference on Research and Development in Information Retrieval (2015), ACM, pp. 413-422.

[31] Ye, M., Janowicz, K., Mülligann, C., and Lee, W.-C. What you are is when you are: the temporal dimension of feature types in location-based social networks. In Proceedings of the 19th ACM SIGSPATIAL International Conference on Advances in Geographic Information Systems (2011), ACM, pp. 102-111.

[32] Ye, M., Yin, P., Lee, W.-C., And Lee, D.-L. Exploiting geographical influence for collaborative point-of-interest recommendation. In Proceedings of the 34th international ACM SIGIR conference on Research and development in Information Retrieval (2011), ACM, pp. 325-334.

[33] Yin, H., Sun, Y., Cui, B., Hu, Z., And Chen, L. Lcars: a location-content-aware recommender system. In Proceedings of the 19th ACM SIGKDD International Conference on Knowledge Discovery and Data mining (2013), ACM, pp. 221-229.
[34] Yin, Z., CaO, L., Han, J., Zhai, C., and Huang, T. Geographical topic discovery and comparison. In Proceedings of the 20th international conference on World wide web (2011), ACM, pp. 247-256.

[35] Zhang, C., Liang, H., Wang, K., and Sun, J. Personalized trip recommendation with poi availability and uncertain traveling time. In Proceedings of the 24th ACM International on Conference on Information and Knowledge Management (2015), ACM, pp. 911-920.

[36] ZhANG, W., AND WANG, J. Location and time aware social collaborative retrieval for new successive point-of-interest recommendation. In Proceedings of the 24th ACM International on Conference on Information and Knowledge Management (2015), ACM, pp. 1221-1230.

[37] Zhao, S., Zhao, T., Yang, H., Lyu, M. R., and King, I. Stellar: Spatial-temporal latent ranking for successive point-of-interest recommendation. In Thirtieth AAAI Conference on Artificial Intelligence (2016).

[38] Zheng, V. W., Zheng, Y., Xie, X., And Yang, Q. Collaborative location and activity recommendations with gps history data. In Proceedings of the 19th International Conference on World Wide Web (2010), ACM, pp. 1029-1038.

[39] Zheng, Y., Zhang, L., Xie, X., And Ma, W.-Y. Mining interesting locations and travel sequences from gps trajectories. In Proceedings of the 18th international conference on World wide web (2009), ACM, pp. 791-800. 